\title{
Classification of Traditional Chinese Medicine Syndromes in Patients with Chronic Hepatitis B by SELDI-Based ProteinChip Analysis
}

\author{
Ya-Nan Song,, Hui Zhang, ${ }^{1}$ Yan Guan, ${ }^{1}$ Jing-Hua Peng, ${ }^{2}$ Yi-Yu Lu, ${ }^{1}$ \\ Yi-Yang $\mathrm{Hu}^{2}$, and Shi-Bing $\mathrm{Su}^{1}$ \\ ${ }^{1}$ Research Center for Traditional Chinese Medicine Complexity System, Shanghai University of Traditional Chinese Medicine, \\ 1200 Cailun Road, Pudong, Shanghai 201203, China \\ ${ }^{2}$ Institute of Liver Diseases, Shuguang Hospital, Key Laboratory of Liver and Kidney Diseases of Ministry of Education, \\ Shanghai University of Traditional Chinese Medicine, Shanghai 201203, China
}

Correspondence should be addressed to Yi-Yang Hu, yyhuliver@163.com and Shi-Bing Su, shibingsu07@163.com

Received 12 January 2012; Accepted 28 March 2012

Academic Editor: Shao Li

Copyright (C) 2012 Ya-Nan Song et al. This is an open access article distributed under the Creative Commons Attribution License, which permits unrestricted use, distribution, and reproduction in any medium, provided the original work is properly cited.

\begin{abstract}
Traditional Chinese medicine (TCM) syndrome, also called ZHENG, is the basis concept of TCM theory. It plays an important role in TCM practice. There are excess and deficiency syndromes in TCM syndrome. They are the common syndromes in chronic hepatitis $\mathrm{B}(\mathrm{CHB})$ patients. Here we aim to explore serum protein profiles and potential biomarkers for classification of TCM syndromes in $\mathrm{CHB}$ patients. 24 healthy controls and two cohorts of $\mathrm{CHB}$ patients of excess syndrome $(n=25)$ or deficiency syndrome $(n=19)$ were involved in this study. Protein profiles were obtained by surface-enhanced laser desorption ionization time-flight mass spectrometry (SELDI-TOF/MS) and multiple analyses were performed. Based on SELDI ProteinChip data, healthy controls and $\mathrm{CHB}$ patients or excess and deficiency syndromes in $\mathrm{CHB}$ patients were obviously differentiated by orthogonal partial least square (OPLS) analysis. Two significant serum proteins ( $\mathrm{m} / \mathrm{z} 4187$ and $\mathrm{m} / \mathrm{z} 5032$ ) for classifying excess and deficiency syndromes were found. Moreover, the area under the receiver operating characteristic (ROC) curve was 0.887 for classifying excess and nonexcess syndrome, and 0.700 for classifying deficiency and nondeficiency syndrome, respectively. Therefore, the present study provided the possibility of TCM syndrome classification in CHB patients using a universally acceptable scientific approach.
\end{abstract}

\section{Introduction}

Traditional Chinese medicine (TCM) syndrome classification (also defined as Zheng differentiation) and treatment is the basis concept of TCM theory. TCM syndrome, a profile of symptoms and signs as a series of clinical phenotypes, plays an important role in understanding the human homeostasis and guiding the applications of Chinese herbs and acupuncture. Heat, cold, excess, and deficiency are the four basic syndromes of maladjustment nature in TCM [1]. Damp heat stasis syndrome and liver and kidney Yin deficiency syndrome, classified as excess syndrome and deficiency syndrome, respectively, are the common syndromes in chronic hepatitis $\mathrm{B}(\mathrm{CHB})$ patients [2]. Excess syndrome refers to the accumulation or stagnation of metabolic waste, body fluids, and blood, whereas deficiency syndrome means to "overcatabolism" and "overconsumption", the deficiency of nutrients, and weakness [1].

So far, an experiential diagnosis approach has been always used to classify excess syndrome and deficiency syndrome in CHB patients. TCM practitioners with rich experience in TCM diagnosis and treatment are often able to improve the symptoms of $\mathrm{CHB}$ patients, which may be considered to be untreatable by conventional medicine [3]. Lu et al. [4] mentioned that for coronary heart patients with different TCM syndromes, if herbal medicine was appropriate to TCM syndrome, the effective rate would increase. It was suggested that syndrome classification acts as a pivot in the therapeutic process and directly affects the therapeutic result of a specific disease. Instead of experiential diagnosis, 
therefore, it is necessary to standardize the diagnosis criteria for classification of excess and deficiency syndromes in patients with CHB by using a universally acceptable scientific approach.

Proteomics, a rapidly evolving tool in systems biology of analyzing protein expression in a comprehensive degree, is widely applied for disease diagnosis and prognosis, such as brain injury [5], appendicitis [6], liver fibrosis [7], and esophageal cancer [8]. Surface-enhanced laser desorption ionization time-flight mass spectrometry (SELDI-TOF/MS), a powerful tool for global analysis of protein expression, provides an efficient and sensitive method for biomarker discovery. It can obtain the spectra composed of hundreds of protein peaks, each characterized by its mass-to-charge ratio $(\mathrm{m} / \mathrm{z})$ and each area represented by its amount [9]. Considering the features of measuring in a high-throughput way and analyzing with a small amount of materials, SELDI-TOF/MS has become an attractive tool for clinical application. The technology has successfully led to the discovery of new biomarkers for diagnosis and treatment of various diseases, for example, accurate diagnosis of early hepatocellular carcinoma [10] and laryngeal carcinoma [11], and identification of treatment efficacy-related host factors in chronic hepatitis $C$ [12].

$\mathrm{CHB}$ is a kind of global infective disease induced by hepatitis virus B (HBV). It is estimated that about 400 million people are suffering from HBV infection worldwide [13, 14]. And HBV leads to 500,000 to 1.2 million deaths every year because of turning into liver cirrhosis and hepatocellular carcinoma (HCC) [15]. With 120 million people infected with HBV, China has the largest population in the world. And among them, about 30 million people are suffering from CHB [3]. TCM is widely used in the treatment of CHB and was found to be effective in China [16-18], and conventional medicine hardly heals $\mathrm{CHB}$ patients completely, so more and more people therefore turn to get help from TCM. In the present study, we aim to use SELDI-TOF/MS analysis and related data processing methods to find the protein profiles of excess and deficiency syndromes and the promising protein biomarkers to classify these TCM syndromes in patients with CHB.

\section{Material and Methods}

2.1. Study Population. The study has been approved by Shuguang Hospital, the affiliated hospital of Shanghai University of TCM. Serum samples were collected from November 2009 to July 2010. The experiment involved 24 healthy controls and two cohorts of CHB patients of excess syndrome $(n=$ $25)$ or deficiency syndrome $(n=19)$. The demographic and clinicopathological data about the participants were showed in Table 1. The differences of gender and age have no statistical significance among three groups $(P>0.05)$. The selected 44 patients with CHB must be in accordance with the following criteria: (1) all patients were diagnosed according to both CHB and TCM syndromes and confirmed by chief physicians; (2) the diagnosis of $\mathrm{CHB}$ was based on the guideline defined by the Chinese Society of Hepatology and Chinese Society of Infectious Diseases in 2005 [19]; (3) the TCM syndrome differentiation was referred to the viral hepatitis diagnostic standard described by the Internal Medicine Hepatopathy Committee of Chinese Traditional Medicine Association in December, 1991 [20]. An informed consent was signed by each of the participants, and the study protocol conformed to the ethical guidelines of the Declaration of Helsinki (1964).

The fasting blood samples were collected from two experimental groups of patients with $\mathrm{CHB}$ and healthy controls in the morning and allowed to stand for $30 \mathrm{~min}$ at room temperature and then centrifuged at $1,5000 \mathrm{rpm}$ for $10 \mathrm{~min}$. All the serum samples were stored at $-80^{\circ} \mathrm{C}$ until further analysis.

\subsection{Protein Profiling by SELDI-TOF/MS. CM10 (Cipher-} gen Biosystems, Fremont, CA, USA) was used to further serum differential protein spectrum analysis. First, $5 \mu \mathrm{L}$ of the cleared serum was mixed with $10 \mu \mathrm{L}$ of U9 solution containing $9 \mathrm{~mol} / \mathrm{L}$ urea, $2 \%$ CHAPS, $50 \mathrm{mmol} / \mathrm{L}$ Tris/ $\mathrm{HCl}$, and 1\% DTT (pH 9.0; Sigma, USA). Subsequently, the previous sample was diluted with $185 \mu \mathrm{L}$ CM10-binding buffer $(50 \mathrm{mmol} / \mathrm{L}$ sodium acetate, $\mathrm{pH} 4.0$; Sigma, USA) to give a final dilution of 40 -fold. In addition, the array spots should be preactivated twice with $200 \mu \mathrm{L}$ of binding buffer for $5 \mathrm{~min}$. And then, $100 \mu \mathrm{L}$ of diluted serum samples was loaded on each array spot and incubated with shaking for $1 \mathrm{~h}$ at $4^{\circ} \mathrm{C}$. Two washes with binding buffer and one quick rinse with HPLC grade water were continued to remove nonselectively bound proteins. After air-drying, $0.5 \mu \mathrm{L}$ of freshly prepared sinapinic acid solution in $0.5 \%$ trifluoroacetic acid and 50\% acetonitrile was added on each spot for twice. The chips were ready for MS detection when dried.

Mass accuracy was calibrated externally by using the allin-one peptide molecular mass standard. After calibration passed, the chips were scanned by SELDI-TOF/MS in a PBSIic ProteinChip reader (Ciphergen Biosystems) to measure the masses and intensities of the protein peaks. According to experience, many parameters were optimized for getting more protein peaks and separating these peaks better. At last, the reader was set up as follows: laser intensity, 100; laser sensitivity, 8; optimized mass range, 2,000-15,000 Da; focus mass, $8,500 \mathrm{Da}$; high mass, $50,000 \mathrm{Da}$; and data acquisition parameters, 25 delta to 5 transients per to 10 ending position to 75 . Data were processed automatically using the Ciphergen Protein-Chip Software (version 3.1.1, Ciphergen Biosystems). Spectra were normalized, calibrated, and aligned.

2.3. Data Processing. Protein spectra were automatically generated after all raw data were collected. The profiling spectra of serum samples were first normalized using total ion current by Ciphergen ProteinChip Software 3.1.1. Peak selection was carried out by the Biomarker Wizard program. Protein peaks were selected based on a first pass of signal-tonoise ratio of 5 . This process was completed with a second pass of signal-to-noise ratio of 2 , and peak selection at 
TABLE 1: Clinical parameters and TCM syndromes in CHB patients and controls.

\begin{tabular}{lccc}
\hline Clinical parameters & Excess syndrome $(n=25)$ & Deficiency syndrome $(n=19)$ & Healthy control $(n=24)$ \\
\hline gender $(\mathrm{M} / \mathrm{F})$ & $21 / 4$ & $14 / 5$ & $15 / 9$ \\
age $($ year $)$ & $38.0 \pm 13.4$ & $38.1 \pm 11.1$ & $36.4 \pm 11.6$ \\
BMI $\left(\mathrm{Kg} / \mathrm{m}^{2}\right)$ & $23.2 \pm 3.0$ & $22.1 \pm 2.7$ & $21.3 \pm 2.1$ \\
ALT $(\mathrm{U} / \mathrm{L})$ & $91.8 \pm 116.8$ & $57.4 \pm 41.7$ & $20.7 \pm 8.7$ \\
AST $(\mathrm{U} / \mathrm{L})$ & $59.8 \pm 54.4$ & $50.5 \pm 29.1$ & $19.9 \pm 5.5$ \\
GGT $(\mathrm{U} / \mathrm{L})$ & $47.8 \pm 47.7$ & $56.8 \pm 72.0$ & $21.5 \pm 9.8$ \\
ALP $(\mathrm{U} / \mathrm{L})$ & $84.2 \pm 21.4$ & $90.2 \pm 34.7$ & $58.0 \pm 20.2$ \\
ALB $(\mathrm{g} / \mathrm{L})$ & $45.2 \pm 4.1$ & $44.2 \pm 3.5$ & $43.9 \pm 5.7$ \\
TG $(\mathrm{mmol} / \mathrm{L})$ & $1.1 \pm 0.4$ & $1.4 \pm 0.7$ & $0.8 \pm 0.3$ \\
BA $(\mu \mathrm{mol} / \mathrm{L})$ & $10.3 \pm 15.9$ & $13.0 \pm 18.2$ & $8.0 \pm 1.8$ \\
TBIL $(\mu \mathrm{mol} / \mathrm{L})$ & $19.8 \pm 8.3$ & $18.9 \pm 5.2$ & $15.0 \pm 3.8$ \\
PT $(\mathrm{s})$ & $13.4 \pm 2.0$ & $13.2 \pm 1.8$ & $12.7 \pm 0.8$ \\
HbsAg $(+/-)$ & $25 / 0$ & $19 / 0$ & $0 / 24$ \\
HBV DNA $(+/-)$ & $19 / 6$ & $11 / 8$ & $0 / 24$ \\
\hline
\end{tabular}

$0.3 \%$ of the mass window, and the estimated peaks were added. After the preliminary analysis of protein spectra, these selected protein peaks were exported to other commercially available software for further analysis.

The statistical analysis was performed by SPSS software (version 15.0, Chicago, IL, USA). Values are expressed as the mean $\pm \mathrm{SD}$. The baseline characteristics were compared using appropriate method. For continuous variables, oneway factorial analysis was used, or the Wilcoxon rank-sum test was used because of the skewed distributions. And for categorical variables, $\mathrm{x}^{2}$ test was used. Multivariate analysis was carried out to determine the independent variables associated with differentiation of syndromes. Two-sided $P$ value $<0.05$ for one-way factorial analysis or adjusted $P$ value $<0.0167$ for Wilcoxon rank-sum test was considered statistically significant. SELDI-TOF/MS-measured variables showing statistical significance on univariate analysis were subjected to binary logistic regression to determine significant independent factors. After the regression, the values of the prediction probability were applied to the classification of the samples. Then receiver operating characteristic curve (ROC) was made by using the SPSS software.

The preprocessed data obtained by Ciphergen ProteinChip Software were also exported and analyzed by principle component analysis (PCA) and orthogonal partial least squares (OPLSs) using the SIMCA-P software (version 11.5, Umetrics AB, Umea, Sweden).

\section{Results}

3.1. Clinical Characteristics of Study Population. Clinical characteristics and TCM syndromes in CHB patients and healthy controls are shown in Table 1. Data including body mass index (BMI), alanine aminotransferase (ALT), aspartate aminotransferase (AST), $\boldsymbol{\gamma}$-Glutamyltransferase (GGT), alkaline phosphatase (ALP), albumin (ALB), triglyceride (TG), bile acid (BA), total bilirubin (TBIL), prothrombin time (PT), Hepatitis B surface antigen ( $\mathrm{HbsAg}$ ), and HBV DNA were expressed as the mean \pm SD. According to the statistical analysis, no clinical factors were significantly different between excess syndrome and deficiency syndrome, indicating that the two TCM syndromes could not been classified by the general clinical parameters of CHB.

3.2. Serum Protein Profiling by SELDI-TOF/MS. Using the SELDI ProteinChip system, we analyzed the serum protein profiling from 24 healthy controls, 25 excess syndrome patients with $\mathrm{CHB}$, and 19 deficiency syndrome patients with CHB. Peaks were detected automatically after baseline subtraction. 184 protein peaks were detected and these peaks were overlapping among 3 groups. Figure 1(a) displays the representative protein profiling obtained by SELDI-TOF/MS analysis showing the protein peaks of healthy controls and $\mathrm{CHB}$ patients of two different TCM syndromes. As shown, the SELDI technology was effective in separating low molecular weight proteins and polypeptides between $\mathrm{m} / \mathrm{z}$ 2,000 and $\mathrm{m} / \mathrm{z} 15,000$.

3.3. Classification of TCM Syndromes by Pattern Recognition Analysis. To explore whether the serum protein profiles could help to classify excess syndrome and deficiency syndrome in $\mathrm{CHB}$ patients, pattern recognition analysis was carried out to analyze the data generated by SELDITOF/MS. Principle component analysis (PCA) was first used as an unsupervised statistical method to study the protein differences among the three groups. The result showed that there was not a trend of separation between control group and $\mathrm{CHB}$ group or excess syndrome and deficiency syndrome groups (Figure 2(a)). Then a supervised statistical method, that is orthogonal partial least squares (OPLSs) analysis, was performed as mentioned before. As OPLS score plots were displayed, a tendency of separation was observed among the three groups (Figure 2(b)), and an obvious separation exists between excess syndrome group and deficiency syndrome group (Figure 2(c)), indicating that the whole protein expression was different not only between 


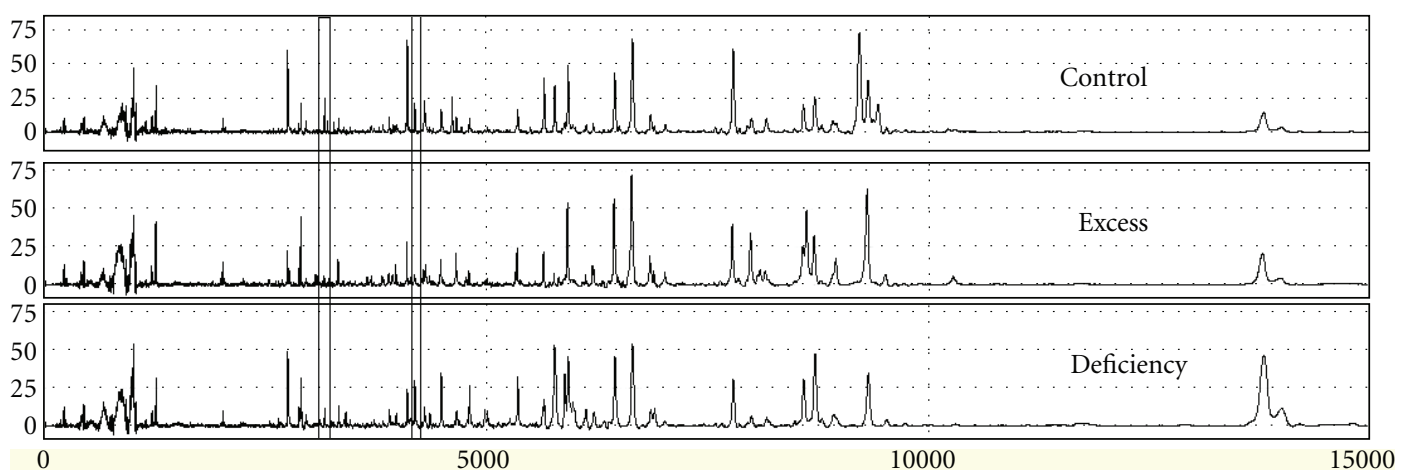

(a)
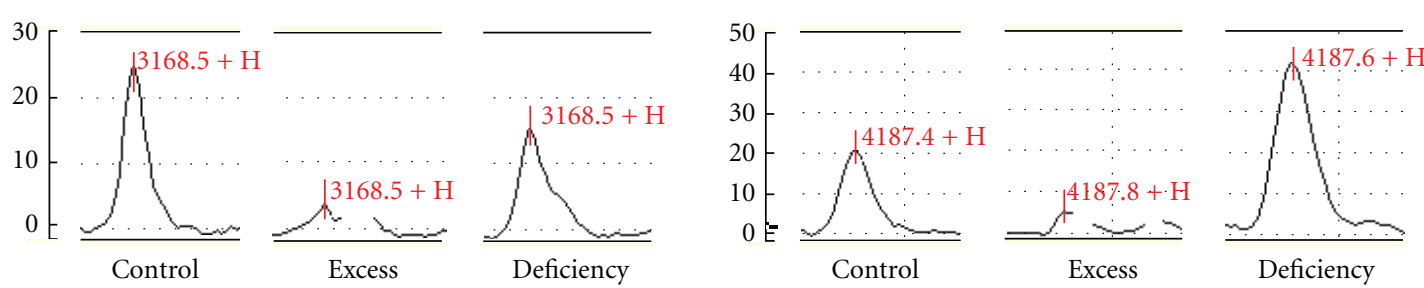

(b)

Figure 1: Representative protein profiles of serum samples of healthy controls and patients with CHB of excess symptom and deficiency syndrome. Protein peak spectrum of serum was analyzed by the SELDI-TOF/MS system, and representative protein peaks within m/z 01,5000 of three groups are shown (a). Statistically significantly different peaks between excess syndrome and deficiency syndrome are shown in the enlarged view, $\mathrm{m} / \mathrm{z} 3168$ on the left and $\mathrm{m} / \mathrm{z} 4187$ on the right (b).

healthy controls and $\mathrm{CHB}$ patients but also between excess and deficiency syndromes in $\mathrm{CHB}$ patients.

On the other hand, to investigate whether clinical parameters had influence on classification, the PCA model comparing three groups was constructed using clinicopathological data alone. But the result was not satisfying and the groups could not be differentiated from each other (not shown). And then the OPLS model was carried out. As shown in Figure 2(d), only the control group could be separated from the two others, whereas the TCM syndrome groups could not be separated from each other. It was suggested that the general clinical data were good at classifying health and HBC, while the data from SELDI-TOF/MS could be used for TCM syndrome classification.

3.4. Serum Protein Potential Biomarkers of TCM Syndromes. Among a total of 184 protein peaks detected, 4 significantly different peaks were observed between excess and deficiency syndromes according to Wilcoxon rank-sum test. Three of four protein peaks were in lower abundance in excess syndrome group (Figures 3(a), 3(b), and 3(c)), and the remaining one was in higher abundance (Figure $3(d)$ ). These statistically significant differences can be displayed clearly in the box-plots. Also, an enlarged view of $\mathrm{m} / \mathrm{z} 3168$ and $\mathrm{m} / \mathrm{z} 4187$ is shown in Figure 1(b). So they may be potential biomarkers for classifying excess syndrome and deficiency syndrome with CHB.

3.5. Logistic Regression Analysis. To identify the variables independently associated with TCM syndromes in CHB patients and to compare the value of SELDI data and clinical parameters in classifying TCM syndromes, logistic regression analysis was performed including SELDI-TOF/MS-measured four significantly different variables displayed in Figure 3 and some clinical parameters listed in Table 1. As shown in Table 2, two protein peaks were independent factors that were associated with TCM syndromes and no clinical parameters were selected. Just as mentioned in Section 3.3, it was proven again that the general clinical data were only good at classifying health and HBC, while the method of SELDITOF/MS could be used for TCM syndrome classification. Then peak m/z 4187 and peak m/z 5032 were applied to the classification of different TCM syndrome. And $88 \%$ of excess syndrome patients and $73.7 \%$ of deficiency syndrome patients were correctly discriminated (cutoff value: 0.5 , Figure 4).

3.6. Sensitivity and Specificity of Serum Protein Markers for TCM Syndrome Classification. To determine the sensitivity and specificity of serum protein potential biomarkers and the usefulness of protein peak quantifications as classification of different TCM syndromes, ROC analysis was conducted. To increase the performance of the classification, the most efficient peak combination was determined using regression analysis. Control group and deficiency syndrome group were put together and defined as the nonexcess syndrome group, so ROC analysis was carried out for discriminating excess syndrome with nonexcess syndrome. The area under the ROC curve for the combination of $\mathrm{m} / \mathrm{z} 4187$ and $\mathrm{m} / \mathrm{z}$ 5032 was 0.887 (Figure 5(a)). In the same way, Control 


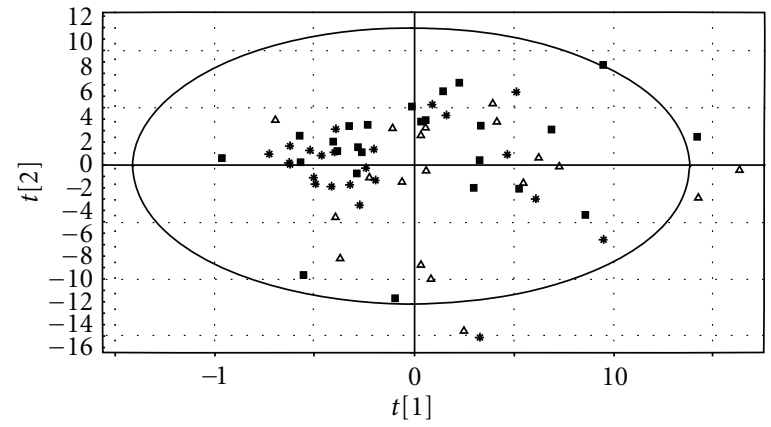

- Excess syndrome

$\triangle$ Deficiency syndrome

* Control

(a)

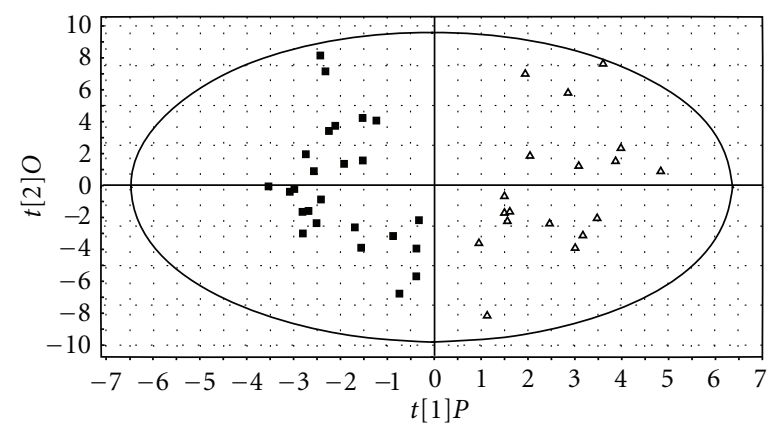

- Excess syndrome

$\Delta$ Deficiency syndrome

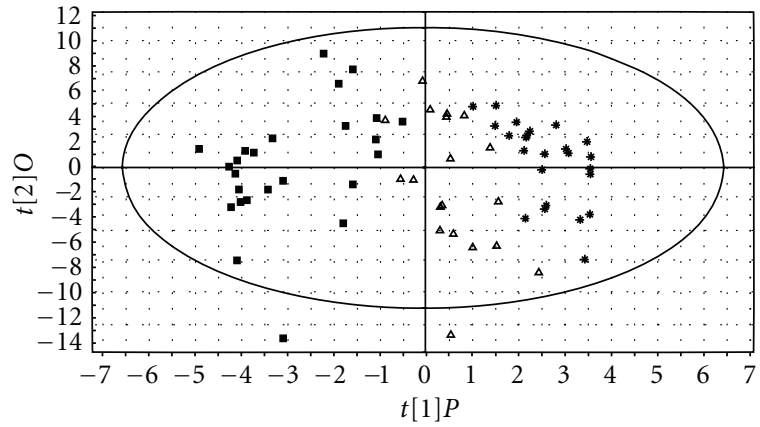

- Excess syndrome

$\Delta$ Deficiency syndrome

* Control

(b)

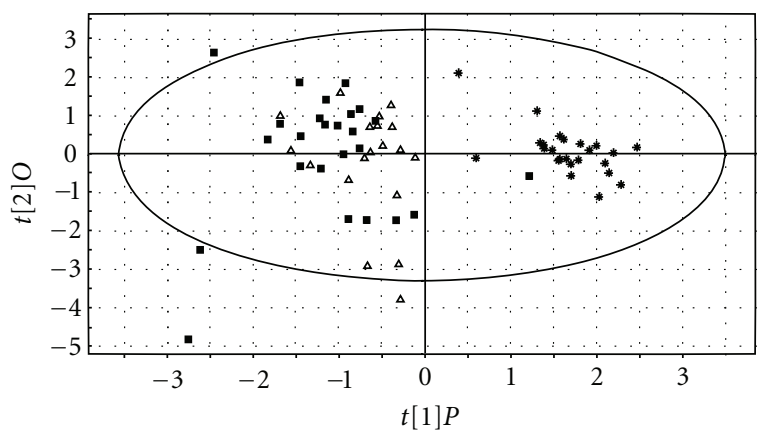

- Excess syndrome

$\triangle$ Deficiency syndrome

* Control

(c)

(d)

Figure 2: PCA score plot and OPLS score plots of $25 \mathrm{CHB}$ patients of excess syndrome $(\mathbf{\square}), 19 \mathrm{CHB}$ patients of deficiency syndrome $(\triangle)$, and 24 healthy controls $(*)$ based on the serum protein profiling detected from SELDI-TOF/MS or the clinicopathological data of each individuals. (a) PCA score plot among the control group and CHB groups of excess syndrome and deficiency syndrome; OPLS score plots (b) among the control group and CHB groups of excess syndrome and deficiency syndrome and (c) between excess syndrome group and deficiency syndrome group. (a)-(c) Models of score plots were constructed by the data from SELDI-TOF/MS. (d) Another OPLS score plot among the three groups using clinical parameters.

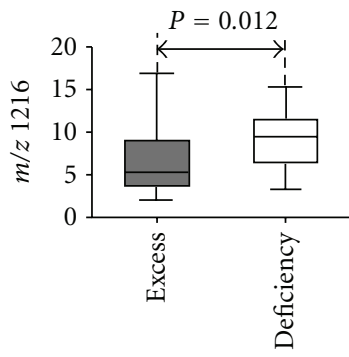

(a)

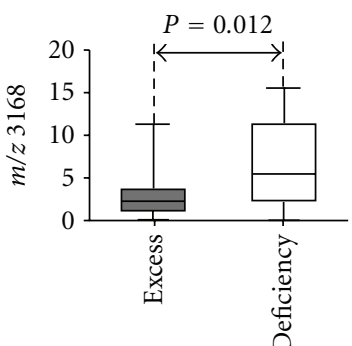

(b)

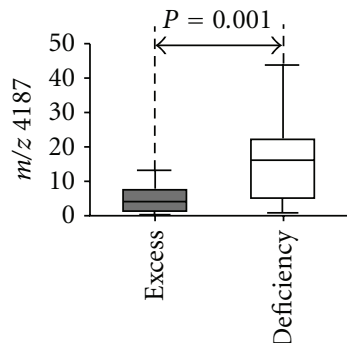

(c)

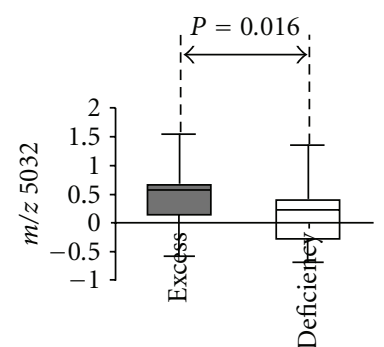

(d)

FIGURE 3: Box-plots for protein peak comparison between TCM syndrome groups. Proteins m/z 1216 (a), m/z 3168 (b), and m/z 4187 (c) were in lower abundance in excess syndrome group than those in deficiency syndrome one, while protein $\mathrm{m} / \mathrm{z} 5032$ (d) was in higher abundance. 
TABLE 2: Logistic regression analysis for TCM syndrome classification in $\mathrm{CHB}$ patients.

\begin{tabular}{lccc}
\hline Factors & Odds ratio & $95 \%$ CI & $P$ value \\
\hline $\mathrm{m} / \mathrm{z} 4187$ & 1.349 & $1.100-1.655$ & 0.004 \\
$\mathrm{~m} / \mathrm{z} 5032$ & 0.054 & $0.005-0.597$ & 0.017 \\
$\mathrm{~m} / \mathrm{z} 1216$ & - & - & 0.894 \\
$\mathrm{~m} / \mathrm{z} 3168$ & - & - & 0.097 \\
$\mathrm{BMI}\left(\mathrm{Kg} / \mathrm{m}^{2}\right)$ & - & - & 0.301 \\
$\mathrm{ALT}(\mathrm{U} / \mathrm{L})$ & - & - & 0.544 \\
$\mathrm{AST}(\mathrm{U} / \mathrm{L})$ & - & - & 0.452 \\
$\mathrm{GGT}(\mathrm{U} / \mathrm{L})$ & - & - & 0.074 \\
$\mathrm{ALP}(\mathrm{U} / \mathrm{L})$ & - & - & 0.779 \\
$\mathrm{ALB}(\mathrm{g} / \mathrm{L})$ & - & - & 0.093 \\
TG $(\mathrm{mmol} / \mathrm{L})$ & - & - & 0.262 \\
$\mathrm{BA}(\mu \mathrm{mol} / \mathrm{L})$ & - & - & 0.206 \\
TBIL $(\mu \mathrm{mol} / \mathrm{L})$ & - & - & 0.901 \\
PT $(\mathrm{s})$ & - & & 0.150 \\
\hline
\end{tabular}

group and excess syndrome group were put together and defined as the nondeficiency syndrome group, and then ROC analysis was performed to discriminate excess syndrome with nonexcess syndrome. The area under the ROC curve was 0.700 (Figure 5(b)). It was suggested that the quantification of these variables by SELDI-TOF/MS was useful to classify excess and deficiency syndromes (Figure 5).

\section{Discussion}

TCM practitioners classify biomedical maladjustments into different syndromes, and each syndrome has its own suitable treatment protocol. Also, considering that the mechanism of disease might not be identical in different people, that is to say, one disease could display several different syndromes, so the same disease may be treated by different therapeutic approaches. The syndrome classification-based individualized therapy is commonly applied in the TCM practice. So we have sufficient reasons to believe that the therapeutic effect will be influenced if excess syndrome and deficiency syndrome of $\mathrm{CHB}$ patients were not classified correctly. Therefore, much attention should be paid to the accuracy and the standard of syndrome classification. However, people often argue that the diagnostic approach of TCM practitioners does not meet requirements of objectivity and reproducibility. And TCM diagnosis studies have proved that there exists considerable variability across different practitioners, even when the same patient was diagnosed $[21,22]$. So it is essential to find a kind of scientific and persuasive approach for the application of TCM syndrome classification.

Proteomics is playing an important role in improving our understanding of biologic systems by observing the different interactions among hundreds of proteins simultaneously and aims at studying proteins of human body in the level of integrity. It happens to be in accordance with the viewpoint of TCM, which has always been emphasized on the integrity of human body and the close relationship between human and its environment [3]. In addition, the characteristics of proteomics make it possible to integrate various proteins [23] and easy to study TCM syndrome classification. Comparing with the traditional method that syndromes are classified into groups based on TCM theory and clinical experiences, they can be clustered into specific groups using the approaches of proteomics and bioinformatics. Matsumoto et al. found several proteins for the diagnosis of "Oketsu", a pathophysiologic concept of Japanese traditional medicine, and differentiated "Oketsu" with "non-Oketsu" successfully [24]. Obviously, it is more scientific and more persuasive. As described in this paper, a proteomics approach was applied, which aimed to provide a kind of accurate and reliable method for TCM syndrome classification.

In this study, we used the ProteinChip system to analyze and compare the serum protein profiles of excess and deficiency syndromes in $\mathrm{CHB}$ patients to define the new potential protein biomarkers for syndrome classification. According to pattern recognition analysis, excess and deficiency syndromes were observed to be clustered into different groups. And four protein peaks were found statistically significant when both groups were compared. On the other hand, syndrome groups could not be classified using general clinical data, and no clinical data were found significantly different between TCM syndrome groups. Among those four possible protein markers, three $(\mathrm{m} / \mathrm{z} 1216, \mathrm{~m} / \mathrm{z} 3168$, and $\mathrm{m} / \mathrm{z} 4187$ ) were overexpressed in the deficiency syndrome group and one $(\mathrm{m} / \mathrm{z} 5032)$ was increased in the group of excess syndrome. Multivariate regression analysis performed by using four significantly different protein peaks from SELDI-TOF/MS data and laboratorial serum markers from clinical data showed the usefulness of two protein peaks (peak m/z 4187 and peak m/z 5032) for excess and deficiency syndromes classification. To observe the sensitivity and specificity of the two proteins, ROC curve analysis was conducted to differentiating excess with nonexcess syndromes and deficiency with nondeficiency syndromes. The area under the ROC curve was 0.887 and 0.700 , respectively, suggesting that they could be applied for the classification of TCM syndromes in CHB patients.

Since one disease could display multiple syndromes in TCM theory, this study focused on several subgroups of $\mathrm{CHB}$ patients. It would make protein profiles of different patients keep in the same level of a specific disease and eliminate the interference of diseases for looking for biomarkers classifying different syndromes.

Also, comparing healthy controls with $\mathrm{CHB}$ patients of excess syndrome or deficiency syndrome, significant variables were supposed to represent the potential biomarkers about $\mathrm{CHB}$ disease and excess syndrome or deficiency syndrome, and the common variables were supposed to represent the potential biomarkers between $\mathrm{CHB}$ and healthy group. So in order to find out potential biomarkers for classifying TCM syndromes, those about CHB disease should be eliminated from the significant variables comparing excess syndrome with deficiency syndrome. Therefore, 27 significantly different serum proteins between healthy controls and excess syndrome might be the potential biomarkers for $\mathrm{CHB}$ disease and excess syndrome. In the same way, 


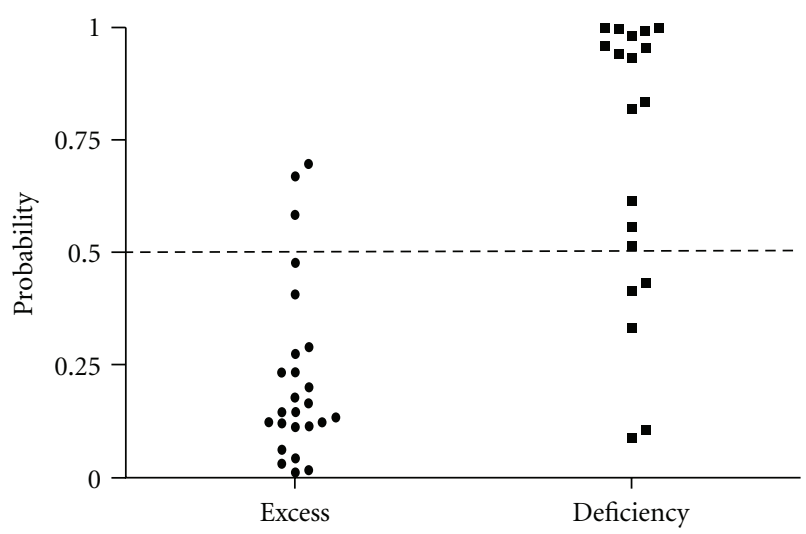

FIGURE 4: Diagnostic potential of the two marker proteins (m/z 4187 and m/z 5032) using binary logistic regression method with the data from different TCM syndromes in CHB patients. $88 \%$ of excess syndrome patients and $73.7 \%$ of deficiency syndrome patients were correctly discriminated (cutoff value: 0.5 ).

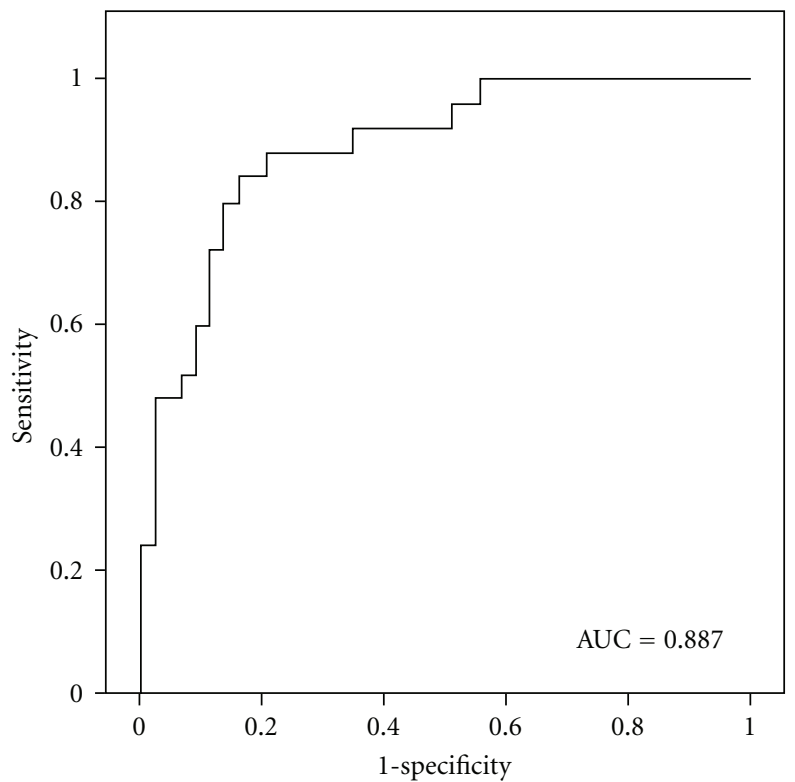

(a)

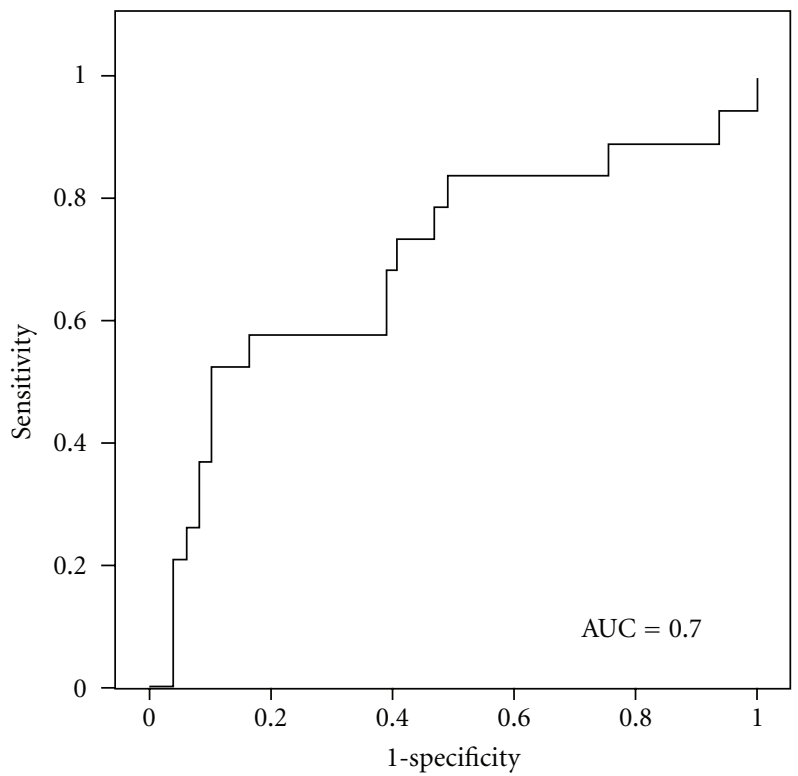

(b)

FIGURE 5: ROC curve for classification of two different TCM syndromes in CHB patients. It was generated combining the peak values of $\mathrm{m} / \mathrm{z}$ 4187 and $\mathrm{m} / \mathrm{z}$ 5032. (a) ROC curve for classification of excess syndrome and non-excess syndrome. AUC (area under the curve) $=0.887$. (b) ROC curve for classification of deficiency syndrome and nondeficiency syndrome. AUC $=0.700$.

28 significantly different ones between healthy controls and deficiency syndrome might be the potential biomarkers for $\mathrm{CHB}$ disease and deficiency syndrome (Table 3). And 9 common proteins (marked in bold in Table 3) were supposed to represent the potential biomarkers between $\mathrm{CHB}$ and healthy group, which should be eliminated from those significantly different proteins between excess syndrome and deficiency syndrome. However, these 9 proteins were totally different with those 4 ones found when comparing between TCM syndrome groups. So it was demonstrated that the interference of diseases to biomarkers had been eliminated.
Most importantly, this study is the first time to classify TCM syndromes in CHB patients by an objective and scientific approach instead of a subjective and experiential one. Our work found the characteristic markers in biochemistry associated with specific TCM syndromes and it will facilitate the development of syndrome classification. Also, it provides an important direction for the understanding and acceptance of TCM theory all around the world. Furthermore, the incorporation of SELDI-based ProteinChip technology into TCM syndrome classification will lead to a new era in the development of TCM to improve treatment efficacy. Our 
TABLE 3: Significantly different peaks between healthy controls and excess or deficiency syndromes. ${ }^{\mathrm{a}}$

\begin{tabular}{|c|c|c|c|c|}
\hline $\mathrm{m} / \mathrm{z}$ & Healthy controls & Excess or deficiency syndrome & Change $^{\mathrm{b}}$ & $P$ value \\
\hline \multicolumn{5}{|c|}{ Healthy control versus excess syndrome } \\
\hline 1174 & $0.20 \pm 0.49$ & $0.69 \pm 1.17$ & $\uparrow$ & 0.011 \\
\hline 2037 & $1.71 \pm 0.71$ & $3.10 \pm 2.24$ & $\uparrow$ & 0.010 \\
\hline 2269 & $0.86 \pm 0.38$ & $1.62 \pm 1.25$ & $\uparrow$ & 0.011 \\
\hline 2592 & $0.49 \pm 0.27$ & $-0.04 \pm 0.34$ & $\downarrow$ & 0.002 \\
\hline 3203 & $2.38 \pm 1.22$ & $0.13 \pm 0.42$ & $\downarrow$ & 0.001 \\
\hline 3408 & $1.71 \pm 0.80$ & $0.21 \pm 0.51$ & $\downarrow$ & 0.000 \\
\hline 4104 & $32.07 \pm 13.66$ & $11.11 \pm 6.43$ & $\downarrow$ & $<0.001$ \\
\hline 4187 & $11.66 \pm 4.61$ & $4.08 \pm 3.33$ & $\downarrow$ & 0.004 \\
\hline 429 & $7.98 \pm 2.88$ & $3.64 \pm 2.53$ & $\downarrow$ & 0.001 \\
\hline 4311 & $4.79 \pm 1.46$ & $1.97 \pm 2.70$ & $\downarrow$ & $<0.001$ \\
\hline 5032 & $-0.05 \pm 0.33$ & $0.48 \pm 0.56$ & $\uparrow$ & $<0.001$ \\
\hline 5497 & $1.75 \pm 0.79$ & $0.37 \pm 0.39$ & $\downarrow$ & $<0.001$ \\
\hline 5650 & $18.09 \pm 6.50$ & $9.10 \pm 5.46$ & $\downarrow$ & 0.001 \\
\hline 7027 & $2.10 \pm 0.75$ & $3.33 \pm 1.97$ & $\uparrow$ & 0.007 \\
\hline 7587 & $1.19 \pm 0.45$ & $2.07 \pm 1.43$ & $\uparrow$ & 0.015 \\
\hline 11732 & $0.52 \pm 0.26$ & $1.04 \pm 0.68$ & $\uparrow$ & 0.001 \\
\hline 14070 & $0.55 \pm 0.23$ & $0.98 \pm 0.65$ & $\uparrow$ & 0.008 \\
\hline 15167 & $1.95 \pm 1.45$ & $4.50 \pm 4.42$ & $\uparrow$ & 0.002 \\
\hline 15354 & $0.49 \pm 0.43$ & $1.22 \pm 1.24$ & $\uparrow$ & 0.002 \\
\hline 22862 & $1.09 \pm 0.63$ & $2.31 \pm 1.47$ & $\uparrow$ & $<0.001$ \\
\hline 23481 & $2.25 \pm 1.36$ & $4.78 \pm 2.65$ & $\uparrow$ & $<0.001$ \\
\hline 28118 & $1.72 \pm 0.65$ & $2.31 \pm 1.02$ & $\uparrow$ & 0.013 \\
\hline 33516 & $0.13 \pm 0.28$ & $0.37 \pm 0.68$ & $\uparrow$ & 0.004 \\
\hline 38571 & $0.04 \pm 0.02$ & $0.08 \pm 0.08$ & $\uparrow$ & 0.005 \\
\hline 38814 & $0.04 \pm 0.02$ & $0.08 \pm 0.08$ & $\uparrow$ & 0.007 \\
\hline 46804 & $0.03 \pm 0.03$ & $0.09 \pm 0.08$ & $\uparrow$ & $<0.001$ \\
\hline 47818 & $0.02 \pm 0.01$ & $0.04 \pm 0.04$ & $\uparrow$ & 0.015 \\
\hline \multicolumn{5}{|c|}{ Healthy control versus deficiency syndrome } \\
\hline 1074 & $0.49 \pm 0.48$ & $0.13 \pm 0.37$ & $\downarrow$ & 0.013 \\
\hline 1210 & $1.89 \pm 1.42$ & $2.64 \pm 1.02$ & $\uparrow$ & 0.006 \\
\hline 1216 & $6.03 \pm 3.50$ & $9.26 \pm 3.39$ & $\uparrow$ & 0.002 \\
\hline 1261 & $21.14 \pm 9.18$ & $29.16 \pm 10.80$ & $\uparrow$ & 0.014 \\
\hline 1440 & $0.79 \pm 1.03$ & $1.36 \pm 0.95$ & $\uparrow$ & 0.004 \\
\hline 2003 & $1.97 \pm 1.04$ & $4.32 \pm 2.74$ & $\uparrow$ & $<0.001$ \\
\hline 2018 & $6.92 \pm 3.50$ & $13.81 \pm 7.86$ & $\uparrow$ & 0.001 \\
\hline 2037 & $1.71 \pm 0.81$ & $3.62 \pm 2.51$ & $\uparrow$ & 0.001 \\
\hline 2269 & $0.86 \pm 0.47$ & $1.93 \pm 1.08$ & $\uparrow$ & $<0.001$ \\
\hline 3331 & $3.78 \pm 2.69$ & $6.43 \pm 3.33$ & $\uparrow$ & 0.006 \\
\hline 4104 & $32.07 \pm 18.72$ & $16.01 \pm 11.01$ & $\downarrow$ & 0.005 \\
\hline 5260 & $0.80 \pm 1.44$ & $1.96 \pm 1.79$ & $\uparrow$ & 0.004 \\
\hline 5346 & $9.05 \pm 13.75$ & $21.53 \pm 15.00$ & $\uparrow$ & 0.007 \\
\hline 5497 & $1.75 \pm 1.37$ & $0.49 \pm 0.50$ & $\downarrow$ & 0.001 \\
\hline 5558 & $0.97 \pm 1.40$ & $1.85 \pm 1.32$ & $\uparrow$ & 0.007 \\
\hline 5650 & $18.09 \pm 10.43$ & $10.39 \pm 7.67$ & $\downarrow$ & 0.015 \\
\hline 5919 & $23.56 \pm 16.61$ & $40.84 \pm 22.76$ & $\uparrow$ & 0.014 \\
\hline 5947 & $2.98 \pm 3.47$ & $6.79 \pm 5.24$ & $\uparrow$ & 0.010 \\
\hline 6128 & $4.83 \pm 5.95$ & $9.72 \pm 6.92$ & $\uparrow$ & 0.003 \\
\hline 8176 & $2.82 \pm 2.70$ & $4.31 \pm 2.73$ & $\uparrow$ & 0.010 \\
\hline 9723 & $0.37 \pm 0.31$ & $0.83 \pm 0.51$ & $\uparrow$ & 0.002 \\
\hline 10292 & $1.17 \pm 0.98$ & $2.40 \pm 1.22$ & $\uparrow$ & 0.001 \\
\hline
\end{tabular}


TABle 3: Continued.

\begin{tabular}{lcccc}
\hline $\mathrm{m} / \mathrm{z}$ & Healthy controls & Excess or deficiency syndrome & Change $^{\mathrm{b}}$ & $\uparrow$ \\
$\mathbf{1 1 7 3 2}$ & $\mathbf{0 . 5 2} \pm \mathbf{0 . 3 6}$ & $\mathbf{0 . 9 0 \pm 0 . 4 2}$ value & $\uparrow$ \\
15009 & $0.07 \pm 0.09$ & $0.60 \pm 1.86$ & $\uparrow .004$ \\
22572 & $0.30 \pm 0.23$ & $0.93 \pm 1.18$ & $\uparrow .002$ \\
$\mathbf{2 2 8 6 2}$ & $\mathbf{1 . 0 9} \pm \mathbf{0 . 6 1}$ & $\mathbf{2 . 3 9 \pm 1 . 3 5}$ & $\uparrow$ & $<0.001$ \\
$\mathbf{2 3 4 8 1}$ & $\mathbf{2 . 2 5} \pm \mathbf{1 . 5 3}$ & $\mathbf{4 . 7 4} \pm \mathbf{2 . 6 2}$ & $\uparrow$ & $<0.001$ \\
$\mathbf{4 6 8 0 4}$ & $\mathbf{0 . 0 3} \pm \mathbf{0 . 0 2}$ & $\mathbf{0 . 0 8} \pm \mathbf{0 . 0 6}$ & $\uparrow .001$ \\
\hline
\end{tabular}

aProtein peaks marked in bold were the common biomarkers for CHB disease. " " $\uparrow$ " and " $\downarrow$ ” represent the protein was up- and downregulated in CHB patients compared with the control, respectively.

researched results also suggest that TCM syndromes really have their own biological fundament.

\section{Conclusion}

The SELDI-based proteomics found some promising protein profiles and potential biomarkers to classify excess and deficiency syndromes in $\mathrm{CHB}$ patients, and it provided an evidence for objective TCM syndrome classification. However, there also exist some limitations in the study, such as the small amount of study population and lack of identification of candidate biomarkers, which would be researched in future study.

\section{Acknowledgments}

This study was supported by the National Science and Technology Major Project of China (nos. 2012ZX10005001-004 and no. 2009ZX09311-003), Leading Academic Discipline Project of Shanghai Municipal Education Commission (no. J50301), and E-Institutes of Shanghai Municipal Education Commission (no. E 03008). Research Center for Traditional Chinese Medicine Complexity System and Shanghai Shuguang Hospital are contributed equally.

\section{References}

[1] W. Y. Jiang, "Therapeutic wisdom in traditional Chinese medicine: a perspective from modern science," Trends in Pharmacological Sciences, vol. 26, no. 11, pp. 558-563, 2005.

[2] Y. Liu, P. Liu, R. Dai et al., "Analysis of plasma proteome from cases of the different traditional Chinese medicine syndromes in patients with chronic hepatitis B," Journal of Pharmaceutical and Biomedical Analysis, vol. 59, no. 1, pp. 173-178, 2012.

[3] W. Xu, L. Q. Su, and H. J. Li, "Research advances and clinical evaluation of drugs for hepatitis B," Chinese Journal of Hospital Pharmacy, vol. 28, no. 9, pp. 737-739, 2008.

[4] A. P. Lu, H. W. Jia, C. Xiao, and Q. P. Lu, "Theory of traditional chinese medicine and therapeutic method of diseases," World Journal of Gastroenterology, vol. 10, no. 13, pp. 1854-1856, 2004.

[5] A. Zuberovic, M. Wetterhall, J. Hanrieder, and J. Bergquist, "CE MALDI-TOF/TOF MS for multiplexed quantification of proteins in human ventricular cerebrospinal fluid," Electrophoresis, vol. 30, no. 10, pp. 1836-1843, 2009.

[6] N. Johannesson, L. Olsson, D. Bäckström, M. Wetterhall, R. Danielsson, and J. Bergquist, "Screening for biomarkers in plasma from patients with gangrenous and phlegmonous appendicitis using CE and CEC in combination with MS," Electrophoresis, vol. 28, no. 9, pp. 1435-1443, 2007.

[7] R. K. T. Kam, T. C. W. Poon, H. L. Y. Chan, N. Wong, A. Y. Hui, and J. J. Y. Sung, "High-throughput quantitative profiling of serum $\mathrm{N}$-glycome by MALDI-TOF mass spectrometry and $\mathrm{N}$ glycomic fingerprint of liver fibrosis," Clinical Chemistry, vol. 53, no. 7, pp. 1254-1263, 2007.

[8] G. Zhou, H. Li, D. DeCamp et al., "2D differential ingel electrophoresis for the identification of esophageal scans cell cancer-specific protein markers." Molecular \& Cellular Proteomics, vol. 1, no. 2, pp. 117-124, 2002.

[9] T. W. Hutchens and T. T. Yip, "New desorption strategies for the mass- spectrometric analysis of macromolecules," Rapid Communications in Mass Spectrometry, vol. 7, no. 7, pp. 576580, 1993.

[10] L. Chen, D. W. Y. Ho, N. P. Y. Lee et al., "Enhanced detection of early hepatocellular carcinoma by serum SELDITOF proteomic signature combined with alpha-fetoprotein marker," Annals of Surgical Oncology, vol. 17, no. 9, pp. 2518$2525,2010$.

[11] C. Liu, C. Pan, H. Wang, and L. Yong, "Effect of surfaceenhanced laser desorption/ionization time-of-flight mass spectrometry on identifying biomarkers of laryngeal carcinoma," Tumor Biology, pp. 1-7, 2011.

[12] N. Fujita, M. Nakanishi, J. Mukai et al., "Identification of treatment efficacy-related host factors in chronic hepatitis $\mathrm{C}$ by proteinchip serum analysis," Molecular Medicine, vol. 17, no. 1-2, pp. 70-78, 2011.

[13] W. F. Leemans, H. L. A. Janssen, and R. A. de Man, "Future prospectives for the management of chronic hepatitis B," World Journal of Gastroenterology, vol. 13, no. 18, pp. 25542567, 2007.

[14] D. Lavanchy, "Worldwide epidemiology of HBV infection, disease burden, and vaccine prevention," Journal of Clinical Virology, vol. 34, no. 1, pp. S1-S3, 2005.

[15] D. C. Valla, R. De Franchis, A. Hadengue et al., "EASL International Consensus Conference on Hepatitis B. 13-14 September, 2002 Geneva, Switzerland. Consensus statement (long version)," Journal of Hepatology, vol. 39, no. S1, pp. S3S25, 2003

[16] S. L. Wang, N. L. Yao, and W. L. Lv, "Advances in studies on effect superiorities of traditional Chinese medicine on chronic hepatitis B," China Journal of Chinese Materia Medicasource, vol. 32, no. 23, pp. 2468-2470, 2007.

[17] L. L. Tang, J. F. Sheng, C. H. Xu, and K. Z. Liu, "Clinical and experimental effectiveness of Astragali compound in the treatment of chronic viral hepatitis B," Journal of International Medical Research, vol. 37, no. 3, pp. 662-667, 2009. 
[18] B. E. Wang, "Treatment of chronic liver diseases with traditional Chinese medicine," Journal of Gastroenterology and Hepatology, vol. 15, pp. E67-E70, 2000.

[19] Chinese Society of Hepatology and Chinese Society of Infectious Diseases, Guide to Prevention and Treatment of Chronic Hepatitis B, 2005.

[20] "The standards of TCM differential syndromes of viral hepatitis," Defined in December 1991 by Internal Medicine Hepatopathy Committee of Chinese Traditional Medicine Association.

[21] C. J. Hogeboom, K. J. Sherman, and D. C. Cherkin, "Variation in diagnosis and treatment of chronic low back pain by traditional Chinese medicine acupuncturists," Complementary Therapies in Medicine, vol. 9, no. 3, pp. 154-166, 2001.

[22] B. Zell, J. Hirata, A. Marcus, B. Ettinger, A. Pressman, and K. M. Ettinger, "Diagnosis of symptomatic postmenopausal women by traditional Chinese medicine practitioners," Menopause, vol. 7, no. 2, pp. 129-134, 2000.

[23] J. F. Cui, Y. K. Liu, H. J. Zhou et al., "Screening serum hepatocellular carcinoma-associated proteins by SELDI-based protein spectrum analysis," World Journal of Gastroenterology, vol. 14, no. 8, pp. 1257-1262, 2008.

[24] C. Matsumoto, T. Kojima, K. Ogawa et al., "A proteomic approach for the diagnosis of 'Oketsu' (blood stasis), a pathophysiologic concept of Japanese traditional (Kampo) medicine," Evidence-Based Complementary and Alternative Medicine, vol. 5, no. 4, pp. 463-474, 2008. 


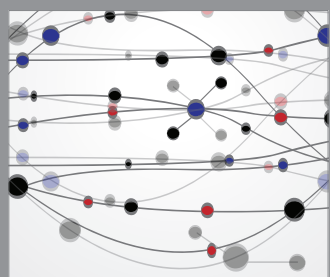

The Scientific World Journal
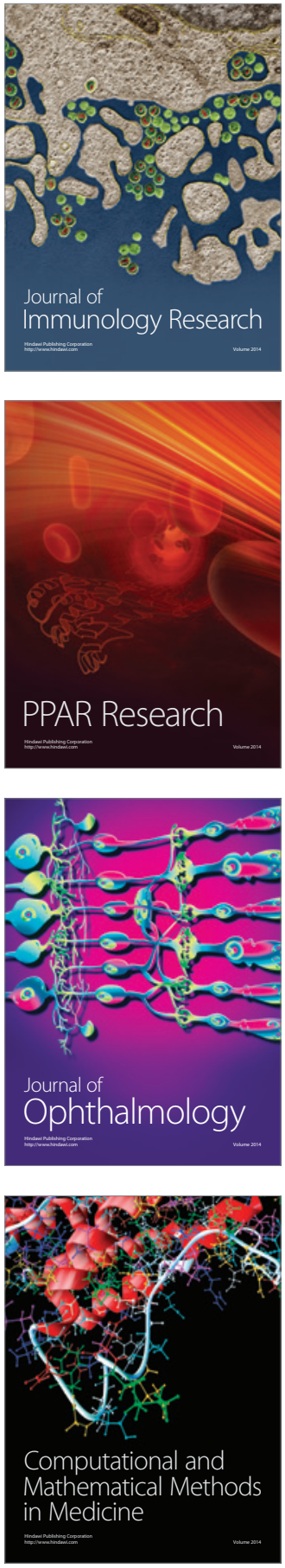

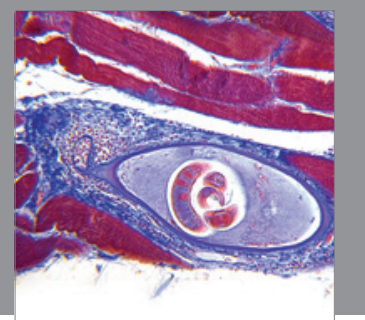

Gastroenterology

Research and Practice
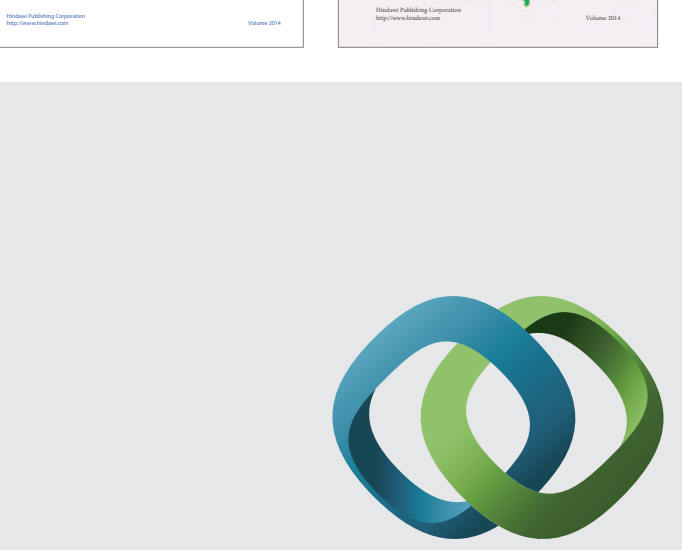

\section{Hindawi}

Submit your manuscripts at

http://www.hindawi.com
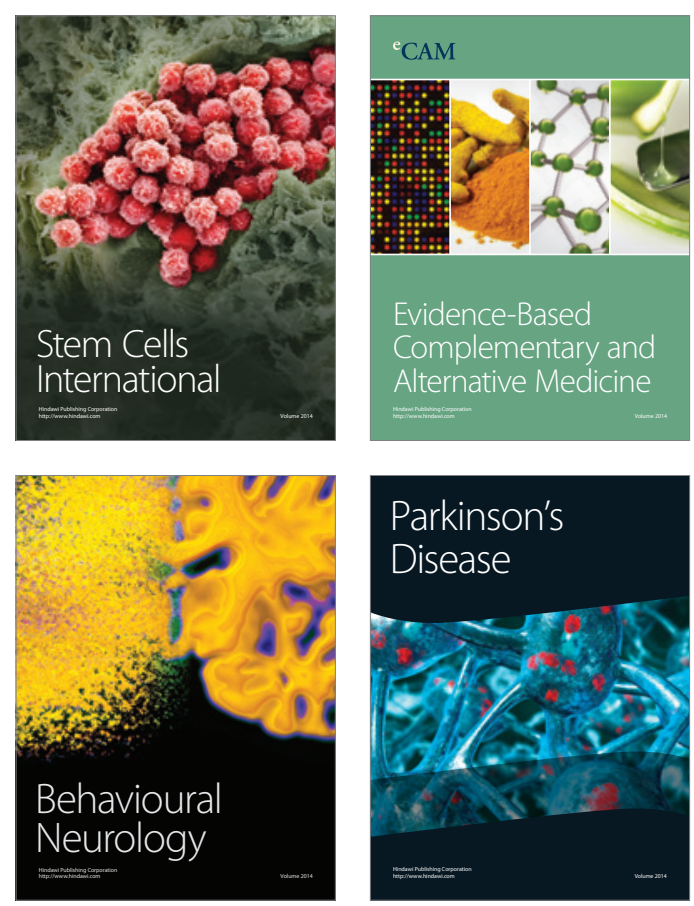

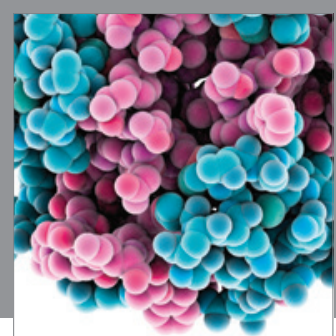

Journal of
Diabetes Research

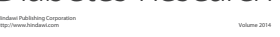

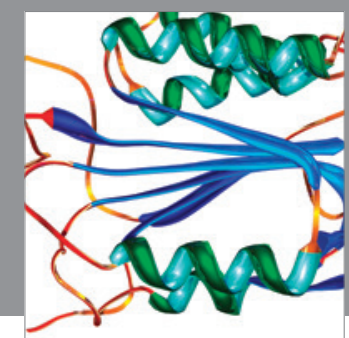

Disease Markers
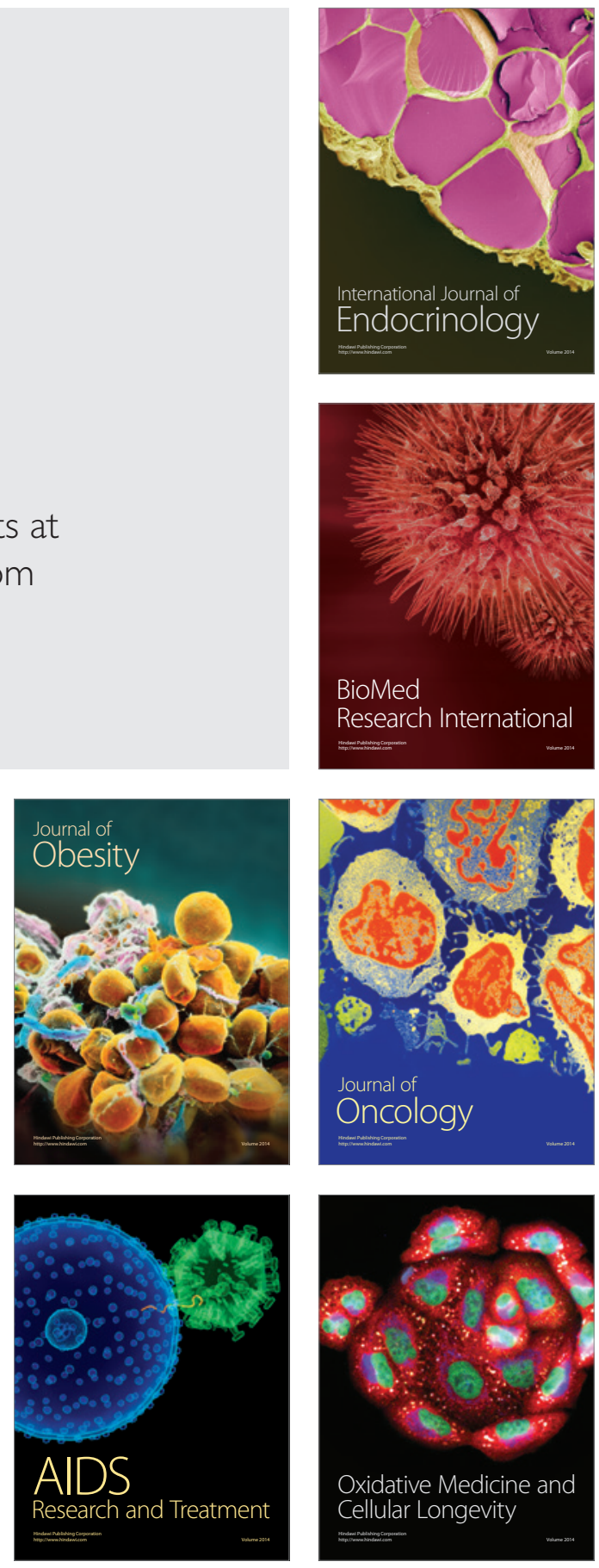